\title{
Gene expression and nutrient utilization in the basideomycete Moniliophthora perniciosa suggest the existence of regulatory mechanisms of catabolite repression
}

\author{
I.S. Ganda
}

2010. Programa de Pós-Graduação em Genética e Biologia Molecular, Universidade Estadual de Santa Cruz (UESC), Ilhéus, BA, Brasil. Extended Abstract of Dissertation. Orienting Prof.: Dr. Cristina Pungartnik.

Moniliophthora perniciosa, a hemibiotrophic fungus (characterized by biotrophic and saprotrophic phases in its life cycle), is the causal agent of witches' broom disease (WBD), which negatively affects the Latin American cocoa (Theobroma cacao) production. The southern region of Bahia, in Brazil, had the first occurrence of WBD in 1989, which led to a great drop in cacao production in the following years. During the biotrophic phase, the infecting fungus promotes stem enlargement, hyperplasic and hypertrophic tissue growth, loss of apical dominance, and green broom formation in T. cacao. During the saprotrophic phase of fungal infection, the green broom turns into a dry one, due to an intense programmed cell death that leads to necrosis of infected parts of the plant. Studies have suggested the presence of a carbon repression (CR) mechanism in M. perniciosa. CR is well characterized in the yeast Saccharomyces cerevisiae and consists in the repression or induction of a set of genes according to the type of carbon source present in the environment. MIG1 and SNF1 genes encode transcription factors that are involved in the regulation of the CR process. The aim of this study was to demonstrate the existence of $\mathrm{CR}$ and to characterize its process in M. perniciosa through physiological studies and analysis of gene expression. The fungus was grown in media with different glucose and glycerol concentrations, and mycelial growth, carbon and peptone consumption profile of the culture medium, $\mathrm{pH}$ variation, and resistance to oxidative stress generated by external hydrogen peroxide $\left(\mathrm{H}_{2} \mathrm{O}_{2}\right)$ were monitored. Real-time polymerase chain reaction (qPCR) was used for expression analysis of selected genes of CR, energy metabolism, autophagy, and protection against oxidative stress agents. The main results were: i) peptone seems to be important in fungal nutrition and growth, being preferentially consumed, even in the presence of glucose or glycerol; ii) growth in medium with glycerol as the main carbon source induced a higher resistance to $\mathrm{H}_{2} \mathrm{O}_{2}$, a slower growth of mycelia and a lower carbon consumption, as compared to glucose-supplemented medium, during 35 days of 
culture; iii) increasing glucose concentrations led to a proportional delay in mycelial growth, a higher resistance to $\mathrm{H}_{2} \mathrm{O}_{2}$ and an increased expression of cytoplasmic catalase-encoding gene $\mathrm{MpCTT1;} \mathrm{iv)} \mathrm{there} \mathrm{was} \mathrm{a} \mathrm{shift} \mathrm{to} \mathrm{alkaline} \mathrm{conditions} \mathrm{(} \mathrm{pH}>8.0)$ in the culture medium during growth of the fungus; v) time of culture of M. perniciosa did not alter the cells' $\mathrm{H}_{2} \mathrm{O}_{2}$ resistance profile; vi) there was no change in the expression of $\mathrm{Mp} P C K$ (phosphoenolpyruvate carboxykinase) and $\mathrm{Mp} P Y K$ (pyruvate kinase) genes; vii) $\mathrm{Mp} P D H$ (pyruvate dehydrogenase) gene expression increased with rising glucose concentration; viii) expression of the transcription factors MpCrel and MpSnfl (here shown for the first time to exist in M. perniciosa) was compatible with a predicted CR mechanism, as we found higher MpCRE1 than MpSNF1 gene expression in the presence of glucose, but this expression was not proportional to glucose concentration as would be expected; ix) sequencing and bioinformatics analysis of MpSNF1 and MpCRE1 PCR products revealed an amino acid identity of 68 and $77.5 \%$ to homologous Snflp and Mig1p sequences of $S$. cerevisiae, respectively. These results show that in a complex medium, peptone is primarily consumed at the expense of a carbon source, but having the choice between glycerol and glucose, the latter is preferred. Considering all the results, the existence of a carbon repression mechanism in M. perniciosa cannot be ruled out. Consumption of glucose or glycerol in M. perniciosa is, however, not regulated in the same manner as in the yeast $S$. cerevisiae.

Key words: Carbon repression; Energy metabolism; Glucose; Glycerol; Peptone 